Geometry $\&$ Topology

Volume 8 (2004) 1032-1042

Published: 7 August 2004

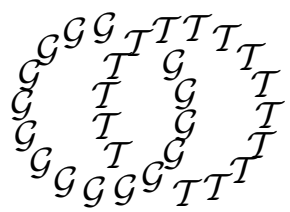

\title{
Weighted $L^{2}$-cohomology of Coxeter groups based on barycentric subdivisons
}

\author{
BORIS L OKUN \\ Department of Mathematical Sciences \\ University of Wisconsin-Milwaukee \\ Milwaukee, WI 53201, USA \\ Email: okun@uwm.edu
}

\begin{abstract}
Associated to any finite flag complex $L$ there is a right-angled Coxeter group $W_{L}$ and a contractible cubical complex $\Sigma_{L}$ (the Davis complex) on which $W_{L}$ acts properly and cocompactly, and such that the link of each vertex is $L$. It follows that if $L$ is a generalized homology sphere, then $\Sigma_{L}$ is a contractible homology manifold. We prove a generalized version of the Singer Conjecture (on the vanishing of the reduced weighted $L_{\mathbf{q}}^{2}$-cohomology above the middle dimension) for the right-angled Coxeter groups based on barycentric subdivisions in even dimensions. We also prove this conjecture for the groups based on the barycentric subdivision of the boundary complex of a simplex.
\end{abstract}

AMS Classification numbers Primary: 58G12

Secondary: 20F55, 57S30, 20F32, 20J05

Keywords: Coxeter group, aspherical manifold, barycentric subdivision, weighted $L^{2}$-cohomology, Tomei manifold, Singer conjecture

Proposed: Benson Farb

Seconded: Walter Neumann, Steven Ferry
Received: 15 March 2004

Revised: 3 August 2004 


\section{Introduction}

A construction of Davis ([1, 2], 4]), associates to any finite flag complex $L$, a "right-angled" Coxeter group $W_{L}$ and a contractible cubical cell complex $\Sigma_{L}$ on which $W_{L}$ acts properly and cocompactly. $W_{L}$ has the following presentation: the generators are the vertices of $L$, each generator has order 2 , and two generators commute if the span an edge in $L$. The most important feature of this construction is that the link of each vertex of $\Sigma_{L}$ is isomorphic to $L$. A simplicial complex $L$ is a generalized homology $m$-sphere (for short, a GHS ${ }^{m}$ ) if it is a homology $m$-manifold having the same homology as a standard sphere $\mathbb{S}^{m}$ (the homology is with real coefficients.) It follows, that if $L$ is a $\mathrm{GHS}^{n-1}$, then $\Sigma_{L}$ is a homology $n$-manifold.

If $L$ is a simplicial complex, $b L$ will denote the barycentric subdivision of $L$. $b L$ is a flag simplicial complex. Let $\partial \Delta^{n}$ denote the boundary complex of the standard $n$-dimensional simplex.

We study a certain weighted $L^{2}$-cohomology theory $L_{\mathbf{q}}^{2} \mathcal{H}^{*}$, described in [7], [5]. Suppose, for each vertex of $v \in L$ we are given a positive real number $q_{v}$, and let q denote the vector with components $q_{v}$. Given a minimal word $w=v_{1} \ldots v_{n} \in$ $W_{L}$, let $\mathbf{q}^{w}=q_{v_{1}} \ldots q_{v_{n}}$. For each $W_{L}$-orbit of cubes pick a representative $\sigma_{0}$ and let $w(\sigma)=w$ if $\sigma=w \sigma_{0}$. (The ambiguity in the choices will not matter in our discussion.) Let $L_{\mathbf{q}}^{2} C^{i}\left(\Sigma_{L}\right)=\left\{\Sigma c_{\sigma} \sigma \mid \Sigma c_{\sigma}^{2} \mathbf{q}^{w(\sigma)}<\infty\right\}$ be the Hilbert space of infinite $i$-cochains, which are square-summable with respect to the weight $\mathbf{q}^{w}$. The usual coboundary operator $d$ is then a bounded operator, and we define the reduced weighted $L_{\mathbf{q}}^{2}$-cohomology to be $L_{\mathbf{q}}^{2} \mathcal{H}^{i}\left(\Sigma_{L}\right)=\operatorname{Ker}\left(d^{i}\right) / \overline{\operatorname{Im}\left(d^{i-1}\right)}$. Similarly, one can define the reduced weighted $L_{\mathbf{q}}^{2}$-homology, except, instead of the usual boundary operator one uses the adjoint of $d$. It follows from the Hodge decomposition that the resulting homology and cohomology spaces are naturally isomorphic. These spaces are Hilbert modules over the Hecke-von Neumann algebra $\mathcal{N}_{\mathbf{q}}$ (an appropriately completed Hecke algebra of $W_{L}$.) This

allows us to introduce the weighted $L_{\mathbf{q}}^{2}$ Betti numbers - the dimension of $L_{\mathbf{q}}^{2} \mathcal{H}^{i}$ over $\mathcal{N}_{\mathbf{q}}$. If $\mathbf{q}=\mathbf{1}=(1, \ldots, 1)$, we obtain the usual reduced $L^{2}$-cohomology, and we omit the index $\mathbf{q}$. We write $\mathbf{q} \leq \mathbf{1}$, if each component of $\mathbf{q}$ is $\leq 1$.

The following conjecture, attributed to Singer, goes back to 1970's.

The Singer Conjecture If $M^{n}$ is a closed aspherical manifold, then

$$
L^{2} \mathcal{H}^{i}\left(\widetilde{M}^{n}\right)=0 \text { for all } i \neq n / 2 .
$$

As explained in [5. Section 14], the appropriate generalization of the Singer Conjecture to the weighted case is the following conjecture: 
The Generalized Singer Conjecture Suppose $L$ is a flag GHS $^{n-1}$. Then $L_{\mathbf{q}}^{2} \mathcal{H}^{i}\left(\Sigma_{L}\right)=0$ for $i>n / 2$ and $\mathbf{q} \leq \mathbf{1}$.

(Poincaré duality shows that for $\mathbf{q}=\mathbf{1}$ this conjecture implies the Singer Conjecture for $\Sigma$.)

This conjecture holds true for $n \leq 4$ by [5]. One of the main results of this paper is a proof of this conjecture for barycentric subdivisions in even dimensions. The proof uses a reduction to a very special case $L=b \partial \Delta^{2 k-1}$.

We prove this case as Theorem 5.2. It turns out (Theorem 5.3), that this result implies the vanishing of the $L_{\mathbf{q}}^{2}$-cohomology in a certain range for arbitrary right-angled Coxeter groups based on barycentric subdivisions. (For $\mathbf{q}=\mathbf{1}$, this implication is proved in [6].) In particular, it follows that the Generalized Singer Conjecture is true for all barycentric subdivisions in even dimensions (Theorem [5.4), and for $b \partial \Delta^{n}$ in all dimensions (Theorem [5.6).

This paper relies very heavily on $[5$. In the inductive proofs we mostly omit the first steps, they are easy exercises using [5].

The author wishes to thank Mike Davis, Jan Dymara and Tadeusz Januszkiewicz for several illuminating discussions.

\section{Vanishing conjectures}

We will follow the notation from [5]. Given a flag complex $L$ and a full subcomplex $A$, set:

$$
\begin{aligned}
\mathfrak{h}_{i}^{\mathbf{q}}(L) & =L^{2} \mathcal{H}_{i}\left(\Sigma_{L}\right) \\
\mathfrak{h}_{i}^{\mathbf{q}}(A) & =L^{2} \mathcal{H}_{i}\left(W_{L} \Sigma_{A}\right) \\
\mathfrak{h}_{i}^{\mathbf{q}}(L, A) & =L^{2} \mathcal{H}_{i}\left(\Sigma_{L}, W_{L} \Sigma_{A}\right) \\
\mathrm{b}_{\mathbf{q}}^{i}(L) & =\operatorname{dim}_{\mathcal{N}_{\mathbf{q}}}\left(\mathfrak{h}_{i}^{\mathbf{q}}(L)\right) \\
\mathrm{b}_{\mathbf{q}}^{i}(L, A) & =\operatorname{dim}_{\mathcal{N}_{\mathbf{q}}}\left(\mathfrak{h}_{i}^{\mathbf{q}}(L, A)\right)
\end{aligned}
$$

The dimension of $\Sigma_{L}$ is one greater than the dimension of $L$. Hence, $\mathrm{b}_{\mathbf{q}}^{i}(L)=0$ for $i>\operatorname{dim} L+1$.

We will use the following three properties of $L_{\mathbf{q}}^{2}$-homology.

Proposition 2.1 (See [5, Section 15]) 
The Mayer-Vietoris sequence If $L=L_{1} \cup L_{2}$ and $A=L_{1} \cap L_{2}$, where $L_{1}$ and $L_{2}$ (and therefore, $A$ ) are full subcomplexes of $L$, then

$$
\rightarrow \mathfrak{h}_{i}^{\mathbf{q}}(A) \rightarrow \mathfrak{h}_{i}^{\mathbf{q}}\left(L_{1}\right) \oplus \mathfrak{h}_{i}^{\mathbf{q}}\left(L_{2}\right) \rightarrow \mathfrak{h}_{i}^{\mathbf{q}}(L) \rightarrow
$$

is weakly exact.

The Künneth Formula The Betti numbers of the join of two complexes are given by:

$$
\mathrm{b}_{\mathbf{q}}^{k}\left(L_{1} * L_{2}\right)=\sum_{i+j=k} \mathrm{~b}_{\mathbf{q}}^{i}\left(L_{1}\right) \mathrm{b}_{\mathbf{q}}^{j}\left(L_{2}\right) .
$$

Poincaré Duality If $L$ is a flag $\operatorname{GHS}^{n-1}$, then $\mathrm{b}_{\mathbf{q}}^{i}(L)=\mathrm{b}_{\mathbf{q}^{-1}}^{n-i}(L)$.

If $\sigma$ is a simplex in $L$, let $L_{\sigma}$ denote the link of $\sigma$ in $L$. To simplify notation we will write $b L_{v}$ instead of $(b L)_{v}$ to denote the link of the vertex $v$ in $b L$. Let $\mathcal{C}$ be a class of GHS's closed under the operation of taking link of vertices, i.e. if $S \in \mathcal{C}$ and $v$ is a vertex of $S$ then $S_{v} \in \mathcal{C}$. Following Section 15 of [5] we consider several variations of the Generalized Singer Conjecture for the class $\mathcal{C}$.

$\mathbf{I}(n) \quad$ If $S \in \mathcal{C}$ and $\operatorname{dim} S=n-1$, then $\mathrm{b}_{\mathbf{q}}^{i}(S)=0$ for $i>n / 2$ and $\mathbf{q} \leq \mathbf{1}$.

$\mathbf{I I I}^{\prime}(2 k+1)$ Let $S \in \mathcal{C}$ and $\operatorname{dim} S=2 k$. Let $v$ be a vertex of $S$. Then the map $i_{*}: \mathfrak{h}_{k}^{\mathbf{q}}\left(S_{v}\right) \rightarrow \mathfrak{h}_{k}^{\mathbf{q}}(S)$, induced by the inclusion, is the zero homomorphism for $\mathbf{q} \geq \mathbf{1}$.

$\mathbf{V}(n) \quad$ Let $S \in \mathcal{C}$ and $\operatorname{dim} S=n-1$. Let $A$ be a full subcomplex of $S$.

- If $n=2 k$ is even, then $\mathrm{b}_{\mathbf{q}}^{i}(S, A)=0$ for all $i>k$ and $\mathbf{q} \leq \mathbf{1}$.

- If $n=2 k+1$ is odd, then $\mathrm{b}_{\mathbf{q}}^{i}(A)=0$ for all $i>k$ and $\mathbf{q} \leq \mathbf{1}$.

The argument in Section 16 of [5] goes through without changes if we consider only GHS's from a class $\mathcal{C}$ to give the following:

Theorem 2.2 (Compare [5, Section 16]) If we only consider GHS's from a class $\mathcal{C}$, then the following implications hold.

(1) $\mathbf{I}(2 k+1) \Longrightarrow \mathbf{I I I}^{\prime}(2 k+1)$.

(2) $\mathbf{V}(n) \Longrightarrow \mathbf{I}(n)$.

(3) $\mathbf{V}(2 k-1) \Longrightarrow \mathbf{V}(2 k)$.

(4) $\left[\mathbf{V}(2 k)\right.$ and $\left.\mathbf{I I I}^{\prime}(2 k+1)\right] \Longrightarrow \mathbf{V}(2 k+1)$. 
Let $\mathcal{J D}$ denote the class of finite joins of the barycentric subdivisions of the boundary complexes of standard simplices:

$$
\mathcal{J D}=\left\{b \partial \Delta^{n_{1}} * \cdots * b \partial \Delta^{n_{i}}\right\}
$$

Lemma 2.3 The class $\mathcal{J D}$ is closed under the operation of taking link of vertices.

Proof Let $S=b \partial \Delta^{n_{1}} * \cdots * b \partial \Delta^{n_{j}}$ and $v \in S$. We can assume that $v \in b \partial \Delta^{n_{1}}$. Then $S_{v}=b \partial \Delta_{v}^{n_{1}} * b \partial \Delta^{n_{2}} * \cdots * b \partial \Delta^{n_{j}}=b \partial \Delta^{\operatorname{dim}(v)} * b \partial \Delta^{n_{1}-\operatorname{dim}(v)-1} * b \partial \Delta^{n_{2}} *$ $\cdots * b \partial \Delta^{n_{j}}$, and therefore $S \in \mathcal{J} \mathcal{D}$.

Next, consider the following statement:

$\mathbf{I I I}^{\prime \prime}(2 k+1)$ Let $v$ be a vertex of $b \partial \Delta^{2 k+1}$. Then the map $i_{*}: \mathfrak{h}_{k}^{\mathbf{q}}\left(b \partial \Delta_{v}^{2 k+1}\right) \rightarrow$ $\mathfrak{h}_{k}^{\mathbf{q}}\left(b \partial \Delta^{2 k+1}\right)$, induced by the inclusion, is the zero homomorphism for $\mathbf{q} \geq \mathbf{1}$.

Lemma 2.4 $\operatorname{III}^{\prime \prime}(2 k+1) \Longrightarrow \mathbf{I I I}^{\prime}(2 k+1)$ for the class $\mathcal{J} \mathcal{D}$.

Proof By induction, we can assume that the lemma holds for all odd numbers $<2 k+1$. Then it follows from the Theorem 2.2 that $\mathbf{V}(m)$ and therefore $\mathbf{I}(m)$ hold for all $m<2 k+1$.

Let $S=b \partial \Delta^{n_{1}} * \cdots * b \partial \Delta^{n_{j}}$ with $n_{1}+\cdots+n_{j}=2 k+1$ and $v \in S$. We assume that $v \in b \partial \Delta^{n_{1}}$. Then $S_{v}=b \partial \Delta_{v}^{n_{1}} * b \partial \Delta^{n_{2}} * \cdots * b \partial \Delta^{n_{j}}$ and, by the Künneth formula, the map in question decomposes as the direct sum of maps of the form

$$
\left(\mathfrak{h}_{k_{1}}^{\mathbf{q}}\left(b \partial \Delta_{v}^{n_{1}}\right) \rightarrow \mathfrak{h}_{k_{1}}^{\mathbf{q}}\left(b \partial \Delta^{n_{1}}\right)\right) \otimes \bigotimes_{i=2}^{j}\left(\mathfrak{h}_{k_{i}}^{\mathbf{q}}\left(b \partial \Delta^{n_{i}}\right) \rightarrow \mathfrak{h}_{k_{i}}^{\mathbf{q}}\left(b \partial \Delta^{n_{i}}\right)\right)
$$

where $k_{1}+\cdots+k_{j}=k$. Since $n_{1}+\cdots+n_{j}=2 k+1$ it follows that $k_{i}<n_{i} / 2$ for some index $i$. If $n_{i}<2 k+1$, then the range of the corresponding map in the above tensor product is 0 by $\mathbf{I}\left(n_{i}\right)$ and Poincaré duality, and therefore the tensor product map is 0 . If $n_{i}=2 k+1$ then, in fact, $i=1$ (the join is a trivial join) and the result follows from III $^{\prime \prime}(2 k+1)$.

Thus, it follows from Theorem 2.2. Lemmas 2.3 and 2.4 and induction on dimension, that in order to prove the Generalized Singer Conjecture for the class $\mathcal{J D}$ all we need is to prove $\mathbf{I I I}^{\prime \prime}(2 k+1)$. 


\section{Removal of an odd-dimensional vertex}

Let $L$ be a simplicial complex and $b L$ be its barycentric subdivision. The vertices of $b L$ are naturally graded by "dimension": each vertex $v$ of $b L$ is the barycenter of a unique cell (which we still denote $v$ ) of the complex $L$, and we call the dimension of this cell the dimension of the vertex $v$. Let $E_{L}$ denote the subcomplex of $b L$ spanned by the even dimensional vertices. Let $\mathcal{A}_{L}$ denote the set of full subcomplexes $A$ of $L$ containing $E_{L}$, which have the following property: if $A$ contains a vertex of odd dimension $2 j+1$, then $A$ contains all vertices of $b L$ of dimensions $\leq 2 j$. In other words, any such $A$ can be obtained inductively from $b L$ by repeated removal of an odd-dimensional vertex of the highest dimension.

If $L=\partial \Delta^{n}$ we will use the notation $E_{n}=E_{L}$ and $\mathcal{A}_{n}=\mathcal{A}_{L}$.

Lemma 3.1 Assume $\mathbf{I I I}^{\prime \prime}(2 m+1)$ holds for $2 m+1<n$. Then for any $(n-1)$-dimensional simplicial complex $L$ and any complex $A \in \mathcal{A}_{L}$ we have:

$$
\mathrm{b}_{\mathbf{q}}^{i}(A)=\mathrm{b}_{\mathbf{q}}^{i}(b L)=0 \text { for } i>(n+1) / 2 \text { and } \mathbf{q} \leq \mathbf{1} .
$$

Proof By induction, we can assume that the lemma holds for all $m<n$. First, we claim that removal of odd-dimensional vertices does not change the homology above $(n+1) / 2$. Let $A \in \mathcal{A}_{L}$ and let $B=A-v$ where $v$ is a vertex of the highest odd dimension of $A$. We let $\operatorname{dim}(v)=2 d-1,1 \leq d \leq k$. We want to prove that $\mathrm{b}_{\mathbf{q}}^{i}(A)=\mathrm{b}_{\mathbf{q}}^{i}(B)$ for $i>(n+1) / 2$. Consider the Mayer-Vietoris sequence of the union $A=B \cup_{A_{v}} C A_{v}$ :

$$
\rightarrow \mathfrak{h}_{i}^{\mathbf{q}}\left(A_{v}\right) \rightarrow \mathfrak{h}_{i}^{\mathbf{q}}(B) \oplus \mathfrak{h}_{i}^{\mathbf{q}}\left(C A_{v}\right) \rightarrow \mathfrak{h}_{i}^{\mathbf{q}}(A) \rightarrow \mathfrak{h}_{i-1}^{\mathbf{q}}\left(A_{v}\right)
$$

Suppose $i>(n+1) / 2$. Since $A_{v}=B \cap b L_{v}=B \cap\left(b \partial \Delta^{2 d-1} * b\left(L_{v}\right)\right)$, and since $B \in \mathcal{A}_{L}$, it follows, by construction, that $A_{v}$ splits as the join:

$$
A_{v}=b \partial \Delta^{2 d-1} * A_{1},
$$

with $A_{1} \in \mathcal{A}_{\left(L_{v}\right)}$. By inductive assumption the lemma holds for $L_{v}$, i.e. $\mathrm{b}_{\mathbf{q}}^{i}\left(A_{1}\right)=\mathrm{b}_{\mathbf{q}}^{i}\left(b\left(L_{v}\right)\right)=0$ for $i>(n+1) / 2-d$.

Since III" $(2 d-1)$ holds by hypothesis, by Lemma 2.4 and Theorem 2.2. $\mathbf{I}(2 d-1)$ holds for the class $\mathcal{J D}$, and, thus, $\mathrm{b}_{\mathbf{q}}^{i}\left(b \partial \Delta^{2 d-1}\right)=0$ for $i \geq d$.

Then, by the Künneth formula, $\mathrm{b}_{\mathbf{q}}^{i-1}\left(A_{v}\right)=0$ for $i-1 \geq(n+1) / 2$, i.e. for $i>(n+1) / 2$. By [5, Proposition 15.2(d)], $\mathrm{b}_{\mathbf{q}}^{i}\left(C A_{v}\right)=\frac{1}{q_{v}+1} \mathrm{~b}_{\mathbf{q}}^{i}\left(A_{v}\right)$. Therefore in the above sequence the terms corresponding to $A_{v}$ and $C A_{v}$ are 0 , and 
the claim follows. Then it follows by induction, that $\mathrm{b}_{\mathbf{q}}^{i}(A)=\mathrm{b}_{\mathbf{q}}^{i}(b L)$ for all $A \in \mathcal{A}_{L}$ and $i>(n+1) / 2$.

To prove the vanishing we note that, in particular, $\mathrm{b}_{\mathbf{q}}^{i}\left(E_{L}\right)=\mathrm{b}_{\mathbf{q}}^{i}(b L)$ for $i>$ $(n+1) / 2$. Since $E_{L}$ is spanned by the even-dimensional vertices of $b L$ and since a simplex in $b L$ has vertices of pairwise different dimensions, we have $\operatorname{dim}\left(E_{L}\right)=[(n+1) / 2]-1$. Therefore, $\mathrm{b}_{\mathbf{q}}^{i}\left(E_{L}\right)=0$ for $i>(n+1) / 2$ and we have proved the lemma.

In the special case $L=\Delta^{2 k+1}$ this lemma admits the following strengthening:

Lemma 3.2 Let $n=2 k+1$. Assume III' $(2 m+1)$ holds for $2 m+1<n$. Then for any complex $A \in \mathcal{A}_{n}, A \subset b \partial \Delta^{n}$, we have:

$$
\mathrm{b}_{\mathbf{q}}^{i}(A)=\mathrm{b}_{\mathbf{q}}^{i}\left(b \partial \Delta^{n}\right) \text { for } i>k \text { and } \mathbf{q} \leq \mathbf{1} .
$$

Proof We proceed as in the previous proof. As before, we have $B=A-v$, $\operatorname{dim}(v)=2 d-1$ and $A_{v}=b \partial \Delta^{2 d-1} * A_{1}$, where now $A_{1} \subset b \partial \Delta^{2 k+1-2 d}$. Therefore, the inductive assumption and the hypothesis on $\mathbf{I I I}^{\prime \prime}(2 d-1)$ imply that $b_{i}\left(A_{1}\right)=0$ for $i>k+d$. The lemma follows as before.

As explained in [6], when $\mathbf{q}=\mathbf{1}$, the removal of the odd-dimensional vertex does not change homology in all dimensions. We record this result below.

Lemma 3.3 Assume III' $(2 m+1)$ holds for $2 m+1<n$ and $\mathbf{q}=\mathbf{1}$. Then for any $(n-1)$-dimensional simplicial complex $L$ and for any complex $A \in \mathcal{A}_{L}$, obtained by the repeated removal of highest odd-dimensional vertices, we have:

$$
\mathrm{b}^{*}(A)=\mathrm{b}^{*}(b L) .
$$

Proof Again we repeat the proof of Lemma 3.1. As before, we have the splitting $A_{v}=b \partial \Delta^{2 d-1} * A_{1}$. The point now is that for $\mathbf{q}=\mathbf{1}, \mathbf{I}(2 d-1)$ and Poincaré duality imply $\mathrm{b}^{*}\left(b \partial \Delta^{2 d-1}\right)=0$ and therefore $\mathrm{b}^{*}\left(A_{v}\right)=0$ by the Künneth formula.

\section{Intersection form}

Lemma 4.1 Let $L$ be a $\mathrm{GHS}^{2 k}$ and let $v$ be a vertex of $L$. Then the image of the restriction map on $L^{2}$-cohomology $i^{*}: L^{2} \mathcal{H}^{k}\left(\Sigma_{L}\right) \rightarrow L^{2} \mathcal{H}^{k}\left(\Sigma_{L_{v}}\right)$ is an isotropic subspace of the intersection form of $\Sigma_{L_{v}}$. 
Proof Note that the cup product of two $L^{2}$-cocycles is an $L^{1}$-cocycle. The intersection form is the result of evaluation of the cup product of two middledimensional cocycles on the fundamental class, which is $L^{\infty}$. Since $\Sigma_{L_{v}}$ bounds a half-space in $\Sigma_{L}, i_{*}\left(\left[\Sigma_{L_{v}}\right]\right)=0$ in $L^{\infty}$-homology of $\Sigma_{L}$. Thus, if $a, b \in$ $L^{2} \mathcal{H}^{n}\left(\Sigma_{L}\right)$, then $\left\langle i^{*}(a) \cup i^{*}(b),\left[\Sigma_{L_{v}}\right]\right\rangle=\left\langle a \cup b, i_{*}\left(\left[\Sigma_{L_{v}}\right]\right)\right\rangle=0$.

Lemma 4.2 Let $G$ be a group and let $A$ be a bounded $G$-invariant (with respect to the diagonal action) non-degenerate bilinear form on a Hilbert submodule $M \subset \ell^{2}(G)$. Then $A$ has no nontrivial $G$-invariant isotropic subspaces.

Proof Let us consider the case $M=\ell^{2}(G)$ first. $G$-invariance and continuity of the form $A$ implies that $A$ is completely determined by it values $a_{g}=(g A 1)$, $g \in G$. It is convenient to think of the form as given by $(x A y)=\langle x, A y\rangle$, where $\langle$,$\rangle is the inner product and A=\Sigma_{g \in G} a_{g} g$ is a bounded $G$-equivariant operator on $\ell^{2}(G)$. Non-degeneracy of $A$ means that $A x=0$ only if $x=0$. $A$ is the limit of the group ring elements, and $A x$ is the limit of the corresponding linear combinations of $G$-translates of $x$, i.e. $A x=\lim \Sigma_{g \in G_{n}} a_{g}(g x)$, where $G_{n}$ is some exhaustion of $G$ by finite sets. It follows that if $x$ belongs to $G-$ invariant isotropic subspace, then $A x$ belongs to the closure of this subspace. Thus, we have $\langle A x, A x\rangle=(A x A x)=0$ by isotropy and continuity, therefore $x=0$.

The case of general submodule $M \subset \ell^{2}(G)$ reduces to the above, since the bilinear form $A$ can be extended to $\ell^{2}(G)$, for example, by taking the orthogonal sum $A \oplus\langle$,$\rangle of A$ on $M$ and the inner product on the orthogonal complement of $M$.

\section{$5 \quad$ Vanishing theorems}

Our main technical results are the following two theorems.

Theorem $\mathbf{5 . 1}$ III $^{\prime \prime}(2 k+1)$ is true for all $k>0$ and $\mathbf{q}=\mathbf{1}$.

Proof The proof is by induction on $k$. Suppose the theorem is true for all $m<k$.

Let $v$ be a vertex of $b \partial \Delta^{2 k+1}$. We need to show that the restriction map $i^{*}: \mathfrak{h}^{k}\left(b \partial \Delta^{2 k+1}\right) \rightarrow \mathfrak{h}^{k}\left(b \partial \Delta_{v}^{2 k+1}\right)$ is the 0 -map.

First let us suppose that $v$ is a vertex of dimension 0 , i.e. a vertex of $\Delta^{2 k+1}$. 
Consider the action of the symmetric group $\mathbf{S}_{2 k+1}$ on $\Delta^{2 k+1}$ which fixes the vertex $v$ and permutes other vertices. This action gives a simplicial action of $\mathbf{S}_{2 k+1}$ on $b \partial \Delta^{2 k+1}$ and therefore, after choosing a base point, lifts to a cubical action of $\mathbf{S}_{2 k+1}$ on $\Sigma_{b \partial \Delta^{2 k+1}}$ stabilizing $\Sigma_{b \partial \Delta_{v}^{2 k+1}}$. Let $G^{\prime}$ be the group of cubical automorphisms of $\Sigma_{b \partial \Delta^{2 k+1}}$ generated by this action and the standard action of $W_{b \partial \Delta^{2 k+1}}$, and let $G$ be the orientation-preserving subgroup of $G^{\prime}$. Similarly, let $G_{v}^{\prime}$ be the group of cubical automorphisms of $\Sigma_{b \partial \Delta_{v}^{2 k+1}}$ generated by this action and the standard action of $W_{b \partial \Delta_{v}^{2 k+1}}$, and let $G_{v}$ be the orientation-preserving subgroup of $G_{v}^{\prime}$.

We claim that, as a Hilbert $G_{v}$-module $L^{2} \mathcal{H}^{k}\left(\Sigma_{b \partial \Delta_{v}^{2 k+1}}\right)$, is a submodule of $\ell^{2}\left(G_{v}\right)$. Note that $b \partial \Delta_{v}^{2 k+1}$ is naturally isomorphic to $b \partial \Delta^{2 k}$.

Using the inductive assumption and Lemma 3.3. we can remove from $b \partial \Delta^{2 k}$ all odd-dimensional vertices without changing the $L^{2}$-cohomology: $\mathfrak{h}^{*}\left(E_{2 k}\right)=$ $\mathfrak{h}^{*}\left(b \partial \Delta^{2 k}\right)$. Since the action of $\mathbf{S}_{2 k+1}$ on $b \partial \Delta_{v}^{2 k+1}=b \partial \Delta^{2 k}$ preserves the dimension of the vertices, we have isomorphism $L^{2} \mathcal{H}^{*}\left(G_{v} \Sigma_{E_{2 k}}\right)=L^{2} \mathcal{H}^{*}\left(\Sigma_{b \partial \Delta}{ }^{2 k}\right)$ as Hilbert $G_{v}$-modules.

The complex $E_{2 k}$ is spanned by the even-dimensional vertices of $b \partial \Delta^{2 k}$, which correspond to the proper subsets of vertices of $\Delta^{2 k}$ of odd cardinality. Thus, the dimension of $E_{2 k}$ is $k-1$, and its top-dimensional simplices are chains $v_{0}<v_{0} v_{1} v_{2}<\ldots<v_{0} \ldots v_{2 k-2}$ of length $k$ of distinct vertices of $\Delta^{2 k}$. Therefore $\mathbf{S}_{2 k+1}$ acts transitively on $(k-1)$-dimensional simplices of $E_{2 k}$ and it follows that $G_{v}$ acts transitively on $k$-dimensional cubes of $G_{v} \Sigma_{E_{2 k}}$. Therefore the space of $k$-cochains is a Hilbert $G_{v}$-submodule of $\ell^{2}\left(G_{v}\right)$, and the claim follows from the Hodge decomposition.

We have, by construction, $G_{v}=\operatorname{Stab}_{G}\left(\Sigma_{b \partial \Delta_{v}^{2 k+1}}\right)$. Then the restriction map $i^{*}: L^{2} \mathcal{H}^{k}\left(\Sigma_{b \partial \Delta^{2 k+1}}\right) \rightarrow L^{2} \mathcal{H}^{k}\left(\Sigma_{b \partial \Delta_{v}^{2 k+1}}\right)$ is $G_{v}$-equivariant and therefore its image is a $G_{v}$-invariant subspace of $L^{2} \mathcal{H}^{k}\left(\Sigma_{b \partial \Delta_{v}^{2 k+1}}\right)$. Since $G_{v}$ acts preserving orientation, the intersection form is $G_{v}$-invariant. By Lemma 4.1 the image is isotropic, thus by Lemma 4.2 it is 0 . Thus, the map $i^{*}: \mathfrak{h}^{k}\left(b \partial \Delta^{2 k+1}\right) \rightarrow$ $\mathfrak{h}^{k}\left(b \partial \Delta_{v}^{2 k+1}\right)=L^{2} \mathcal{H}^{k}\left(W_{b \partial \Delta^{2 k+1}} \Sigma_{b \partial \Delta_{v}^{2 k+1}}\right)$ is the 0 -map.

For vertices of the other even dimensions the argument is similar. If $\operatorname{dim}(v)=$ $2 d$, then its link is $b \partial \Delta^{2 d} * b \partial \Delta^{2 k-2 d}$. Again, using Lemma 3.3 we remove, without changing the $L^{2}$-cohomology, all odd-dimensional vertices from each factor to obtain $E_{2 d} * E_{2 k-2 d}$. The group $\mathbf{S}_{2 d+1} \times \mathbf{S}_{2 k-2 d+1}$ acts naturally on $b \partial \Delta^{2 k+1}$ fixing the vertex $v$ and stabilizing both the link and $E_{2 d} * E_{2 k-2 d}$. This action is again transitive on the top-dimensional simplices of $E_{2 d} * E_{2 k-2 d}$, and the rest of the argument goes through. 
Finally, if $v$ is an odd-dimensional vertex, $\operatorname{dim}(v)=2 d+1$, then we have $b \partial \Delta_{v}^{2 k+1}=b \partial \Delta^{2 d+1} * b \partial \Delta^{2 k-2 d-1}$. The hypothesis on $\mathbf{I I I}^{\prime \prime}$ and Theorem 2.2 and Lemma 2.4 imply that both $\mathbf{I}(2 d+1)$ and $\mathbf{I}(2 k-2 d-1)$ hold. Therefore, by the Künneth formula $\mathfrak{h}^{k}\left(b \partial \Delta_{v}^{2 k+1}\right)=0$ in this case.

Theorem 5.2 The Generalized Singer Conjecture holds true for $b \partial \Delta^{2 k+1}$ :

$$
\mathrm{b}_{\mathbf{q}}^{i}\left(b \partial \Delta^{2 k+1}\right)=0 \text { for } i>k \text { and } \mathbf{q} \leq \mathbf{1} .
$$

Proof We proceed by induction on $k$. Using the inductive assumption and Lemma 3.2 we can remove all odd-dimensional vertices without changing the weighted $L_{\mathbf{q}}^{2}$-homology above $k$. Thus, since the remaining part $E_{2 k+1}$ is $k-$ dimensional, the problem reduces to showing that $\mathfrak{h}_{k+1}^{\mathbf{q}}\left(E_{2 k+1}\right)=0$ for $\mathbf{q} \leq \mathbf{1}$. Since $E_{2 k+1}$ is $k$-dimensional, the natural map $\mathfrak{h}_{k+1}\left(E_{2 k+1}\right) \rightarrow \mathfrak{h}_{k+1}^{\mathbf{q}}\left(E_{2 k+1}\right)$ is injective and the result follows from the Theorem 5.1 .

Next, we list some consequences. Lemma 3.1 implies:

Theorem 5.3 Let $b L$ be the barycentric subdivision of an $(n-1)$-dimensional simplicial complex $L$. Then

$$
\mathrm{b}_{\mathbf{q}}^{i}(b L)=0 \text { for } i>(n+1) / 2 \text { and } \mathbf{q} \leq \mathbf{1} .
$$

Taking $L$ to be a $\mathrm{GHS}^{2 k-1}$, we obtain:

Theorem 5.4 The Generalized Singer Conjecture holds true for the barycentric subdivision of a $\mathrm{GHS}^{n-1}$ for all even $n$.

For odd $n$ we obtain a weaker statement:

Theorem 5.5 Let $b L$ be the barycentric subdivision of a $\mathrm{GHS}^{2 k}$. Then

$$
\mathrm{b}_{\mathbf{q}}^{i}(b L)=0 \text { for } i>k+1 \text { and } \mathbf{q} \leq \mathbf{1} .
$$

In particular,

$$
\mathrm{b}^{i}(b L)=0 \text { for } i \neq k, k+1
$$

Specializing further, and combining with Theorem [5.2, we obtain:

Theorem 5.6 The Generalized Singer Conjecture holds true for $b \partial \Delta^{n}$ :

$$
\mathrm{b}_{\mathbf{q}}^{i}\left(b \partial \Delta^{n}\right)=0 \text { for } i>n / 2 \text { and } \mathbf{q} \leq \mathbf{1},
$$

and, therefore, for the class $\mathcal{J D}$. 
Finally, let us mention an application of the above result to a more analytic object. Let $T_{n}$ denote the space of all symmetric tridiagonal $(n+1) \times(n+1)-$ matrices with fixed generic eigenvalues, the so-called Tomei manifold. It is proved in [8] that $T_{n}$ is an $n$-dimensional closed aspherical manifold.

Theorem 5.7 The Singer Conjecture holds true for Tomei manifolds $T_{n}$.

Proof The space $T_{n}$ can be identified with a natural finite index orbifoldal cover of $\Sigma_{b \partial \Delta^{n}} / W_{b \partial \Delta^{n}}$ [3]. Thus $\Sigma_{b \partial \Delta^{n}}$ is the universal cover of $T^{n}$, and the claim follows from the previous theorem.

\section{References}

[1] M W Davis, Groups generated by reflections and aspherical manifolds not covered by Euclidean space, Ann. of Math. 117 (1983) 293-324 MathReview

[2] M W Davis, Coxeter groups and aspherical manifolds, from: "Algebraic topology (Aarhus 1982)", Lecture Notes in Math. 1051, Springer, New York (1984) 197-221 MathReview

[3] M W Davis, Some aspherical manifolds, Duke Math. J. 55 (1987) 105-139 MathReview

[4] M W Davis, Nonpositive curvature and reflection groups, from: "Handbook of Geometric Topology", (R Daverman, R Sher, editors), Elsevier, Amsterdam (2002) 373-422 MathReview

[5] MW Davis, T Januszkiewicz, J Dymara, BL Okun, Weighted $L^{2}-$ cohomology of Coxeter groups, arXiv:math.GT/0402377

[6] M W Davis, B L Okun, $\ell_{2}$-homology of Coxeter groups based on barycentric subdivisions, Topol. Appl. 140 (2004) 197-202

[7] J Dymara, Thin buildings, preprint

[8] C Tomei, The topology of isospectral manifolds of tridiagonal matrices, Duke Math. J. 51 (1984) 981-996 MathReview 